\title{
CAUSAS SOCIAIS E AS NOVAS PLATAFORMAS DE COMUNICAÇÃO: TEORIAS, VIABILIDADE E PRÁTICAS
}

\author{
Causas sociales y las nuevas plataformas de comunicación: \\ Teorías, viabilidad y prácticas
}

\section{Social causes and new communication platforms: Theories, feasibility and practices}

\author{
Leandro Leonardo Batista ${ }^{1}$ \\ Nathalia Santos Andrijic ${ }^{2}$
}

\begin{abstract}
Resumo
O presente projeto busca analisar como causas sociais e suas mensagens podem ser relacionadas às novas plataformas de comunicação, como as mídias sociais. Investiga teorias a esse respeito, práticas de mercado e a viabilidade da relação em prol da melhor comunicação dessas mensagens, explorando suas vantagens e desvantagens. Revela, por fim, que a abordagem do Marketing se mostrou mais benéfica do que o contrário.
\end{abstract}

Palavras-chave: causas sociais, mídias sociais, marketing social.

\begin{abstract}
This study seeks to analyze how social causes and their messages can be related to the new communication platforms, such as social media. It investigates theories about this topic, market practices and the feasibility of the relation in favor of the best communication of these messages, exploring its perks and disadvantages. It reveals, in the end, that the Marketing approach showed itself more beneficial than the opposite.
\end{abstract}

Keywords: social causes, social media, social marketing.

\footnotetext{
${ }^{1}$ Doutor em Comunicação Social pela University of North Carolina e Mestre em Propaganda pela University of North Carolina. Professor Doutor da ECA - USP junto a graduação em Publicidade e ao PPGCOM. Líder do GEPEC - Grupo de Estudos e Pesquisas dos Efeitos da Comunicação da USP. E-mail: leleba@usp.br

${ }^{2}$ Estudante de Publicidade e Propaganda na ECA/USP. E-mail:nahh22@ yahoo.com.br
} 
Causas sociais e as novas plataformas de comunicação: Teorias, viabilidade e práticas de Leandro Leonardo Batista e Nathalia Santos Andrijic

\section{Resumen}

Este proyecto busca analizar cómo causas sociales y sus mensajes pueden estar relacionadas con las nuevas plataformas de comunicación, tales como las redes sociales. Investiga teorías al respecto, las prácticas de mercado y la viabilidad de la relación por el bien de una mejor comunicación de estos mensajes, la exploración de sus ventajas y desventajas. Revela, por último, que el enfoque de lo Marketing era más beneficioso que no.

Palabras-clave: causas sociales, redes sociales, marketing social.

\section{INTRODUÇÃO}

Este projeto se propõe a realizar um estudo das causas sociais nas novas plataformas de comunicação para compreender sua viabilidade e como essas plataformas podem contribuir para a divulgação e interação com algumas destas causas.

Parte-se do pressuposto de que o ambiente virtual é muito propício à divulgação de causas sociais por facilitar as correntes entre amigos, o compartilhamento, o envolvimento, a identificação com ideais entre muitas outras coisas. Nesse espaço demonstra-se possível também a divulgação de causas e conceitos de maneira rápida, interativa e que atinja muitas pessoas, espalhando-se tanto quanto maior for o número de pessoas envolvidas, o que se mostra benéfico para as causas sociais, principalmente as que precisam de grande adesão para efetivamente acontecerem.

Um campo explorado neste trabalho observa se as causas sociais podem ser tratadas como marcas em alguns casos, i.e., um tratamento mais comercial e, em caso afirmativo como isso poderia ajudar na sua divulgação; se o envolvimento com marcas e produtos pode ser equiparado ao envolvimento com as causas e também se o contato com as mensagens nas redes leva as pessoas a participarem de alguma forma mais efetiva, no sentido comportamental, ou seja, saírem do mundo virtual e levarem efetivamente a ação aquilo que apoiam virtualmente, levando a ideologia para as ruas, para o contato real, para ações mais práticas.

A importância do estudo das causas sociais (ex. doação de sangue e órgãos, prevenção contra acidentes e doenças ou participar em doações de agasalhos ou em manifestações políticas) nas redes sociais explora o ponto, foco deste projeto, da disputa pela atenção dessas mensagens em meio a outras tantas tão diversas de marcas e amigos que geram maior interesse e, obtida esta 
Causas sociais e as novas plataformas de comunicação: Teorias, viabilidade e práticas de Leandro Leonardo Batista e Nathalia Santos Andrijic

atenção, como elas podem desfrutar dos benefícios de espaços tão interativos e próximos dos usuários.

O capítulo 2, "Novas plataformas de comunicação: a abertura de novas possibilidades", compreende o embasamento teórico do estudo e trata da Internet e das redes sociais em uma revisão de literatura para compreender essas plataformas, seus usos e a relação com as marcas. $\mathrm{O}$ capítulo 3, "Causas sociais: Definições, complicações e a abordagem do marketing", traz a revisão de literatura também para esta parte do artigo, buscando o aprofundamento nos termos e trazendo a abordagem do marketing no tratamento delas. Ainda nesse capítulo, ocorre a integração entre o que foi visto no primeiro para entender os benefícios das novas plataformas para as causas sociais, buscando o entendimento do estado arte desta área que se desenvolve rapidamente e oferece novos meios e métodos de investigação que geram outras formas de observar o fenômeno e sua integração com o mundo chamado de "off line". No capítulo 4, "Pesquisa exploratória: exemplos da integração entre causas sociais e as novas plataformas", são estudados diferentes casos de comunicação de causas em que as plataformas digitais permitiram a ampliação do movimento. O capítulo 5, "Teoria e prática: análise da pesquisa exploratória”, traz a união entre o arcabouço teórico do estudo e os casos avaliados para desenvolver o que pôde ser aprendido com os exemplos. Na última parte, "Considerações finais" são traçadas uma revisão geral de tudo o que foi visto, as conclusões do estudo e perspectivas futuras.

\section{NOVAS PLATAFORMAS DE COMUNICAÇÃO: A ABERTURA DE NOVAS POSSIBILIDADES}

$\mathrm{Na}$ última década presenciou-se uma explosão de novas plataformas de comunicação baseadas na transmissão de mensagens pelo meio eletrônico. Novos grupos, redes e formas de se comunicar alteraram, inclusive, vários aspectos do comportamento do consumidor e, consequentemente, das empresas e marcas. As novas possibilidades desses canais estão propiciando novas formas de relacionamento, o que já está sendo percebido e aproveitado pelas marcas. A promoção das causas sociais, por outro lado, ainda está em um estágio bastante embrionário no aproveitamento desse cenário.

\subsection{Entendendo as mídias sociais e seus usos}


Causas sociais e as novas plataformas de comunicação: Teorias, viabilidade e práticas de Leandro Leonardo Batista e Nathalia Santos Andrijic

O grande palco das interações no meio digital são as chamadas Mídias Sociais. "'Social media' may be conceptualized as a group of Internet-based applications that build on the ideological and technological foundations of Web 2.0, which allows creation, and exchange of user generated content" (KAPLAN; HAENLEIN, 2010: 61).

Segundo essa definição, o trunfo das Mídias Sociais se encontra na participação ativa dos usuários, os quais podem criar e trocar conteúdos entre si, o que se transforma em uma teia colaborativa. Outra aspecto é o que seria considerado como a extensão da tradicional comunicação por "boca-a-boca" (MANGOLD, FAULDS, 2009). Ao invés de falar apenas para os mais próximos, as pessoas agora têm o poder de comunicar algo a milhares de pessoas com apenas alguns cliques e receber imediatamente um feedback. O conceito de word of mouse ou eWOMs é essencial para entender a dinâmica desses canais. Shu-Chuan Chu, Yoojung Kim (2011: 48) trazem uma referência de Hennig-Thurau et al. (2004: 39) que definem eWOM como "any positive or negative statement made by potential, actual, or former customers about a product or company, which is made available to a multitude of people and institutions via the Internet".

Algumas das maiores vantagens das Mídias Sociais seriam, então, a amplitude e a criação de uma comunicação de duas mãos com o desenvolvimento do diálogo e a rapidez na interação (BIÇAKÇI, 2012).

O maior objetivo das comunidades que se formam nessas plataformas é o compartilhamento, seja de informações, conteúdos ou até mesmo interesses.

Content communities exist for a wide range of different media types, including text (e.g., BookCrossing, via which 750,000+ people from over 130 countries share books), photos (e.g., Flickr), videos (e.g., YouTube), and PowerPoint presentations (e.g., Slideshare). (KAPLAN; HAENLEIN, 2010: 61).

2.2 Mídias Sociais para marcas: mudanças nas relações com consumidores e entre eles mesmos

Muito do comportamento do consumidor tem sido influenciado pela introdução das novas mídias em seu cotidiano.

They have become a major factor in influencing various aspects of consumer behavior including SIGNOS DO CONSUMO, SÃO PAULO - V.7, N.2, 2015. P. 157-175, DEZ. 2015 
Causas sociais e as novas plataformas de comunicação: Teorias, viabilidade e práticas de Leandro Leonardo Batista e Nathalia Santos Andrijic

awareness, information acquisition, opinions, attitudes, purchase behavior, and post-purchase communication and evaluation. (MANGOLD, FAULDS, 2009: 358)

As marcas estão aprendendo a lidar com essas mudanças para reforçar os laços com os consumidores. Se justamente o diálogo é uma das vantagens das Mídias Sociais, eles estão interagindo com as marcas por meio da criação de conteúdos e histórias sobre elas. Essas plataformas estão sendo usadas para gerar experiências e engajamento, o que demanda mais do que simplesmente anúncios estáticos, o conteúdo entregue deve ser ele mesmo engajador e interessante para a audiência.

Por meio da mensuração de dados é possível identificar mais facilmente exatamente aquilo que mais interessa aos públicos da marca dependendo de diversos fatores. Respostas podem ser mensuradas e avaliadas para que mensagens mais assertivas e adequadas às expectativas dos públicos sejam criadas para que as marcas tenham um relacionamento mais íntimo com os consumidores

As Mídias Sociais permitem um contato direto com os consumidores a custos relativamente baixos e níveis de eficiência mais altos do que os que podem ser atingidos com ferramentas tradicionais, o que as torna relevantes não apenas para grandes empresas, mas também para as médias e pequenas até para organizações não-governamentais e sem fins lucrativos. Votações e jogos online, por exemplo, abrem a possibilidade das marcas falarem de seus produtos e seus benefícios de uma maneira mais divertida e engajadora. "Products that are fun, intriguing, highly visible, easy to use, and which engage the emotions are more likely to stimulate conversation than products that do not meet these criteria" (DOBELE, LINDGREEN, BEVERLAND, VANHAMME, \& VAN WIJK, 2007; DOBELE, TOLEMAN, \& BEVERLAND, 2005 apud MANGOLD, FAULDS, 2009: 363).

Primeiro, portanto, as Mídias Sociais possibilitam às marcas falarem com seus consumidores, conversando e trocando informações. Segundo, aos consumidores falarem com outros consumidores. É natural que as pessoas gostem de se comunicar com outras que tenham desejos e interesses similares aos delas. As marcas podem aumentar esse desejo e propiciar canais de troca ao criarem comunidades com indivíduos de interesses semelhantes (MANGOLD, FAULDS, 2009).

Para as corporações é cada vez mais necessário ter seu espaço nessa formação e criar novos métodos para se relacionar com seus consumidores, o que não é uma tarefa tão simples, 
Causas sociais e as novas plataformas de comunicação: Teorias, viabilidade e práticas de Leandro Leonardo Batista e Nathalia Santos Andrijic

mas os ganhos se mostram compensatórios. "Using Social Media is not an easy task and may require new ways of thinking, but the potential gains are far from being negligible." (KAPLAN; HAENLEIN, 2010: 61).

As marcas querem estar onde seus consumidores estão e o fato delas estarem prestando muita atenção nas Mídias Sociais mostra que seu uso não é apenas uma moda, mas sim uma mudança de paradigma (BIÇAKÇI, 2012) em termos de relacionamento e interação.

\section{CAUSAS SOCIAIS: DEFINIÇÕES, COMPLICAÇÕES E A ABORDAGEM DO MARKETING}

Além de produtos e serviços, outro setor da sociedade que pode tirar proveito das novas possibilidades de comunicação oferecidas pelas Mídias Sociais é o das causas sociais. Para entender como isso ocorre, um caminho mostrou-se proveitoso em diversos estudos: traçar paralelos entre o marketing de produtos e o marketing social.

"Marketing social" foi originalmente definido como "the design, implementation, and control of programs calculated to influence the acceptability of social ideas and involving considerations of product planning, pricing, communications and marketing research" (KOTLER e ZALTMAN, 1971: 5). Assim, o marketing social seria a aplicação dos conceitos e técnicas do marketing para o marketing de ideias e causas sociais ao invés de produtos e serviços no sentido comercial, o que tem demonstrado diversos benefícios. Kotler e Zaltman (1971) apresentam o campo do marketing social como um marketing sem fins lucrativos que inclui campanhas de saúde pública (por exemplo, redução de fumo, alcoolismo, uso de drogas, obesidade), campanhas ambientais (por exemplo, proteção da vida selvagem, reciclagem) e outras campanhas (por exemplo, direitos humanos, igualdade social). Eles defendem a existência de ao menos 4 elementos que são adicionados à abordagem social com o uso das perspectivas do marketing para essas campanhas.

O primeiro elemento é a sofisticada pesquisa de marketing para aprender sobre o mercado e a efetividade das alternativas de abordagem. O segundo é o desenvolvimento de novos produtos já que os "marketeiros" sociais podem considerar produtos existentes ou potenciais para tornar mais fácil a adoção do comportamento desejado, um exemplo poderia ser o uso de aparelhos que diminuem automaticamente o aquecimento em residências durante a noite, reduzindo assim o 
Causas sociais e as novas plataformas de comunicação: Teorias, viabilidade e práticas de Leandro Leonardo Batista e Nathalia Santos Andrijic

consumo de energia. O terceiro elemento adicionado pelo marketing social é o uso de incentivos para ir além da composição de mensagens, oferecendo-os para aumentar o nível de motivação, sendo esses incentivos os mais variados, como produtos e recompensas materiais. O quarto elemento é a facilitação, pois ainda que as pessoas desejem alterar seu comportamento precisam investir tempo e esforço e precisam de maneiras para facilitar a adoção desse novo comportamento.

Já em 1952 Wiebe apresentava o fatídico questionamento: "Why can't you sell brotherhood and rational thinking like you sell soap?". A adoção desse paralelo já tradicional nos estudos sobre a comunicação de causas sociais possibilita a compreensão de diversas dimensões do assunto.

Para Manrai e Gardner (1992)

Social ideas are similar to products in that both involve consumption and need satisfaction. For social ideas, consumption involves taking a position on a social issue or adoption of a social cause and need fulfillment involves resolution of a social problem. As suggested by these similarities, product marketing and social idea marketing share a common underlying philosophy.

Apesar de identificarem essa base determinante comum, as autoras traçam ainda 8 diferenças essenciais entre ideias sociais e produtos convencionais. Para o presente estudo, é preciso manter essas diferenças em mente para que não se caia no erro de tratar tudo como se fosse uma coisa só.

Tab. 1 - Adaptação das 8 diferenças entre causas sociais e produtos convencionais de MANRAI, Lalita A.; GARDNER, Meryl P. em Consumer Processing of Social Ideas Advertising: a Conceptual Model.

\begin{tabular}{|l|l|}
\hline DIFERENÇAS & PROPOSIÇÕES \\
\hline 1. Benefícios compartilhados: & $\begin{array}{l}\text { Proposição 1: a percepção dos consumidores de } \\
\text { benefícios individuais (em comparação com os } \\
\text { benefícios sociais) seria menor para ideias sociais do } \\
\text { que para os produtos convencionais; }\end{array}$ \\
\hline 2. Responsabilidades compartilhadas: & $\begin{array}{l}\text { Proposição 2: a percepção dos consumidores de } \\
\text { responsabilidade individual (em comparação com a } \\
\text { social) seria menor para ideias sociais do que para os } \\
\text { produtos convencionais; }\end{array}$ \\
\hline 3. Benefícios retardados: & \begin{tabular}{l} 
Proposição 3: percepção dos consumidores sobre o \\
\hline
\end{tabular} \\
\hline
\end{tabular}


Causas sociais e as novas plataformas de comunicação: Teorias, viabilidade e práticas de Leandro Leonardo Batista e Nathalia Santos Andrijic

\begin{tabular}{|c|c|}
\hline & $\begin{array}{l}\text { tempo dentro do qual benefícios do consumo serão } \\
\text { realizados seria maior para ideias sociais do que para os } \\
\text { produtos convencionais; }\end{array}$ \\
\hline 4. Controlabilidade: & $\begin{array}{l}\text { Proposição 4: percepção dos consumidores de seu } \\
\text { controle sobre o uso e as consequências do consumo } \\
\text { seria menor para ideias sociais do que para os produtos } \\
\text { convencionais; }\end{array}$ \\
\hline 5. Intangibilidade: & $\begin{array}{l}\text { Proposição 5: percepção dos consumidores da } \\
\text { tangibilidade das ideias sociais seria menor do que a de } \\
\text { produtos convencionais; }\end{array}$ \\
\hline 6. Complexidade: & $\begin{array}{l}\text { Proposição 6: percepção dos consumidores da } \\
\text { complexidade de mensagens seria maior para as ideias } \\
\text { sociais do que para os produtos convencionais; }\end{array}$ \\
\hline 7. Reversibilidade: & $\begin{array}{l}\text { Proposição 7: percepção dos consumidores sobre a } \\
\text { reversibilidade de uma decisão de consumo será menor } \\
\text { para as ideias sociais do que para os produtos } \\
\text { convencionais; }\end{array}$ \\
\hline 8. Contra-pressões: & $\begin{array}{l}\text { Proposição 8: os consumidores terão contra-pressões } \\
\text { (pontos de vista opostos) mais intensas nas decisões } \\
\text { sobre a ideia social do que nas decisões sobre os } \\
\text { produtos convencionais. }\end{array}$ \\
\hline
\end{tabular}

Por todos esses fatores, é possível perceber que as barreiras que precisam ser ultrapassadas na comunicação de causas sociais são maiores do que aquelas dos produtos convencionais. As autoras Manrai e Gardner (1992) desenvolvem o desafio apresentado para a comunicação relacionando esse 8 pontos de diferença com 3 aspectos do processamento da exposição a ideias de mensagens sociais: atenção, compreensão e afeição.

Os 4 primeiros pontos - benefícios compartilhados, responsabilidade compartilhada, benefícios retardados e controlabilidade - afetam a atenção para as mensagens. Para isso, a comunicação pode enfatizar contra-pontos relacionados a eles: a ligação entre a sociedade e o indivíduo, a importância de cada um para que algo aconteça, mostrar recompensas e restaurar o controle do indivíduo sobre a situação. Para trabalhar as barreiras causadas pela intangibilidade e complexidade, as mensagens podem tornar as causas mais tangíveis e simples de serem compreendidas. Quanto às respostas afetivas, a reversibilidade e as contra-pressões podem ser endereçadas pela comunicação ao diminuir a associação de sentimentos desagradáveis com o 
Causas sociais e as novas plataformas de comunicação: Teorias, viabilidade e práticas de Leandro Leonardo Batista e Nathalia Santos Andrijic

compromisso, enfatizando o alto benefício sobre a relação de custo-benefício e reconhecer as contra-pressões ao estabelecer o ponto de vista defendido.

\subsection{Causas sociais nas Mídias Sociais}

Se para as marcas há inúmeros benefícios em utilizar as novas mídias, promotores das causas sociais também podem encontrar vantagens para quebrar as barreiras levantadas em torno das causas.

O espaço das novas mídias permite compartilhamento, colaboração e inclusão de conteúdo gerado pelo usuário. Então, em vez de simplesmente receber mensagem, é possível se engajar em uma conversa que gera conteúdo. Os participantes são ativos ao invés de recebedores passivos. Como o consumidor está sempre no coração do processo do marketing social (HASTINGS e HAYWOOD, 1991) na Web 2.0 eles estão no coração de um aspecto específico a estratégia promocional (HANSON, MCKENZIE, NEIGER e THACKERAY, 2008). O que os usuários encontram é um conteúdo gerado por outros usuários, o que pode, por exemplo, diminuir a "contra-pressão" (MANRAI e GARDNER, 1992).

\section{PESQUISA EXPLORATÓRIA: EXEMPLOS DA INTEGRAÇÃO ENTRE CAUSAS SOCIAIS E AS NOVAS PLATAFORMAS}

O objetivo aqui é explorar selecionadas campanhas de causas sociais e problemas de risco que tiveram sucesso nas novas plataformas, buscando estabelecer uma relação entre os desenvolvimentos teóricos do campo e casos reais, o que pode ajudar a entender melhor esta relação.

Os 2 casos estudados se utilizaram justamente das novas plataformas para expandir sua mensagem. Buscou-se compreender essa aplicação e as especificidades das implicações nas mensagens das causas sociais.

Eles foram escolhidos por se tratarem de diferentes causas não possuírem um patrocinador, podendo-se analisar seus desempenhos independentemente de ligações com marcas. No capítulo seguinte, tratar-se-á de traçar um comparativo entre os casos a partir do embasamento teórico dos primeiros capítulos desse projeto. 
Causas sociais e as novas plataformas de comunicação: Teorias, viabilidade e práticas de Leandro Leonardo Batista e Nathalia Santos Andrijic

\subsection{Dumb ways to die}

A campanha contra morte por acidentes com trens começou na Austrália e logo viralizou pelo mundo. Feita pela agência McCANN Melbourne, a campanha partiu de uma grande verdade sobre mortes na linha do trem, segundo ela própria: "Trains travel in a straight line. If you get hit by a train, you've probably done something wrong which makes getting hit by a train one of the dumbest ways to die."

Não são muitas as agências que permitem a si mesmas esse tipo de honestidade quando tratam da campanha de um cliente, mas o cliente Metro e a agência McCANN o fizeram e esse foi o ponto inicial para o sucesso. A estratégia promocional era salientar uma falha do indivíduo em dar atenção a um problema pessoal, dar saliência ao problema.

Segundo o próprio site da agência (http://www.mccann.com.au/project/dumb-ways-todie/), ao invés de um anúncio de serviço público tipicamente sério, a McCANN optou por uma mistura de humor pouco frequente, uma melodia cativante e uma coleção de personagens animados amigáveis para lançar a mensagem como um vídeo de música on-line sob o título convincente 'Dumb Ways to Die'. Ainda segundo o mesmo site oficial, dentro de 24 horas de seu lançamento em 16 de Novembro de 2012, a canção alcançou o top 10 do iTunes e foi classificada como número 6 na categoria cantor / compositor na parada mundial do iTunes apenas 48 horas depois.

Em fevereiro de 2014, o vídeo tem mais de 60 milhões de visualizações e 4 milhões de compartilhamentos e foi apoiado por uma campanha de publicidade integrada, que gerou uma infinidade de paródias globais e continuações com mais de 30 milhões de visualizações combinadas, atraindo enorme atenção da mídia mundial para os trens do metrô e de sua mensagem de segurança.

O aplicativo do jogo chegou ao número 1 em 17 países, incluindo os EUA, Reino Unido, Canadá e Austrália, com mais de 14 milhões de downloads em 135 países. Dumb Ways to Die foi a campanha mais premiada na história do Festival de Criatividade de Cannes (com 28 Leões, incluindo cinco Grands Prix).

Mais importante ainda, 14 milhões de pessoas declararam sentirem-se mais seguras em torno de trens por causa da campanha, e o cliente Metro Trains notou uma redução de $21 \%$ nos 
Causas sociais e as novas plataformas de comunicação: Teorias, viabilidade e práticas de Leandro Leonardo Batista e Nathalia Santos Andrijic

acidentes e mortes na sua rede.

\subsubsection{Dumb ways to die - Como funcionou}

Tudo começou com a decisão em criar entretenimento ao invés da tradicional publicidade, segundo o Diretor Executivo de Criação da McCANN, Mr. Mescall, em entrevista ao AdAge (DIAZ, 2013): "It was the counterintuitive nature of the idea, the weirdness and positivity of the execution, the sheer joy of the song and the video, and the attention to detail across all elements of the work that ensured its success." Como foi visto, "Products that are fun, intriguing, highly visible, easy to use, and which engage the emotions are more likely to stimulate conversation than products that do not meet these criteria" (DOBELE, LINDGREEN, BEVERLAND, VANHAMME, \& VAN WIJK, 2007; DOBELE, TOLEMAN, \& BEVERLAND, 2005 apud MANGOLD, FAULDS, 2009: 363). As ações de Dumb ways to die se encaixavam exatamente nesses critérios, tendo um lado forte de entretenimento ao divulgar a mensagem de segurança, mexendo com as emoções sem apelar para clichês.

O filme principal lançado online tinha personagens adoráveis e amorfos em uma melodia cativante - peças-chave da estratégia de promoção da viralidade que se realizou por meio do compartilhamento do vídeo. O sucesso de Dumb ways to die deveu-se, portanto, em grande parte ao que seria caracterizado como eWOM (HENNIG-THURAU et al., 2004), já que o público

espalhou os filmes, jogos e ações entre seus contatos nas redes sociais, ampliando seu alcance sem o uso de mídia paga por parte do Metrô para tais feitos numéricos.

A longevidade do projeto foi outro fator importante para o seu sucesso. Em maio, a McCANN lançou ainda uma versão do aplicativo local estendendo a longevidade da campanha. Desde o seu lançamento, o aplicativo foi baixado mais de 18 milhões de vezes. O vídeo não foi a única semente viral. De acordo com o site da agência, esta também fez ativações locais ao trazer os personagens ao ar livre com cartazes ousados e exibir nas ruas ambientes que eram feitos para serem compartilhados no Instagram e nas mídias sociais. Os transeuntes podiam tirar fotografias ao lado de personagens da campanha e pressionar um simbólico botão gigante para assumir o compromisso de serem mais cautelosos em torno de trens. A agência também recorreu a uma ferramenta de ensino para crianças na forma de um livro - estendendo o alcance às salas de aula e não apenas em Victoria, na Austrália, mas em todo o mundo. 
Causas sociais e as novas plataformas de comunicação: Teorias, viabilidade e práticas de Leandro Leonardo Batista e Nathalia Santos Andrijic

O que fez a campanha ser um enorme sucesso foi, por fim, a facilidade para ser compartilhada e discutida. Com as plataformas de mídias sociais, foi muito simples permitir que conversas fossem realizadas em alta velocidade e com grande alcance. Estas, em última análise, fizeram com que a jornada do consumidor ao ter contato com as ideias da campanha fosse muito mais significativa, uma vez que o que se objetivava era uma continua lembrança, i.e., a forte presença mental do alerta de proteção em relação às linhas dos trens que foi facilitada pela interação.midiática.

\subsection{Virginia Blood Services}

Em escalas menores também é possível perceber as redes sociais sendo essenciais para campanhas sociais. Um caso de doação de sangue em Virgínia, nos Estado Unidos, é um exemplo.

Para entender esse caso, é preciso primeiro entendê-lo como sendo um Dilema Social, conceito apresentado por Wiener e Doescher (1991: 38). "In a social dilemma, a person who contributes to the community's good receives fewer personal benefits than one who does not, and all group members receive more personal benefits if all contribute than if all do not." Desta forma, diferente do caso anterior, trata-se de um problema social, de pouca saliência para o indivíduo.

O case da instituição Virginia Blood Services tem sido replicado em blogs como exemplo de ação em Social Media que foi local e está sendo eficiente para alcançar as metas do projeto. Diferentemente de Dumb Ways to Die, que tinha escala global, é uma iniciativa de uma instituição menor e local que lida com influenciadores e redes locais para avisar as pessoas sobre a necessidade de doar sangue.

Para Rothschild (1979 apud WIENER e DOESCHER, 1991: 41) é preciso primeiro identificar o motivo pelo qual as pessoas não estão agindo: "scholars who seek design strategies for 'selling brotherhood' should first identify the reasons why people will not act in a pro-social way and then design strategies to overcome those barriers." Foi exatamente assim que as ações para a Virginia Blood Services se desenvolveram. A razão identificada é de que as pessoas precisam ser avisadas sobre a falta de sangue para que se lembrem de doar. Quando são avisadas por alguém que conhecem e confiam a mensagem se torna mais atraente e aumenta a motivação 
Causas sociais e as novas plataformas de comunicação: Teorias, viabilidade e práticas de Leandro Leonardo Batista e Nathalia Santos Andrijic

para doar sangue.

\subsubsection{Virginia Blood Services - Como funcionou}

Para conseguir mais doações, a estratégia está principalmente pautada em influenciadores locais. O pessoal da Virginia Blood Services está explorando as redes sociais para construir relacionamentos com as pessoas na área de Richmond, na qual a instituição atua. É uma estratégia baseada em influenciadores, aqueles que possuem um grande número de seguidores locais, bem como os atuais doadores de sangue. Eles contactaram essas pessoas e pediram para que se tornassem embaixadores da marca, se comunicando com as suas redes quando surgisse uma emergência de necessidade crítica de sangue, criando essencialmente uma "blood lifeline".

$\mathrm{Na}$ entrevista concedida por um representante da instituição (http://www.rickwhittington.com/blog/vbs-social-media-case-study/), ele explica que havia 2 ferramentas para a atuação das ações: a primeira é uma comunidade online chamada VBS Sangue Lifeline (www.BloodLifeline.com) onde as pessoas podem ir para agendar uma doação de sangue, verificar as necessidades de estoque de sangue ou se registrar para doar. Uma vez cadastrado, os inscritos na Lifeline podem receber alertas via SMS, e-mail ou telefone quando seu tipo sanguíneo estiver em falta; a segunda ferramenta é um aplicativo do Facebook chamado Ydonate, o qual permite a base de doadores existentes agendar sua doação no Facebook e, em seguida, compartilhá-la com seus amigos convidando-os a fazê-lo também.

Essa estratégia trabalhou fortemente com 2 aspectos vistos como diferenças essenciais entre ideias sociais e produtos convencionais (MANRAI; GARDNER, 1992). Se "a percepção dos consumidores de seu controle sobre o uso e as consequências do consumo seria menor para ideias sociais do que para os produtos convencionais", os produtos online criados para a campanha fazem com que o senso de controlabilidade seja maior entre os doadores de sangue, já que há noção da necessidade da doação local e a segurança vinculada à instituição do destino do sangue a quem precisa. Ainda, se "os consumidores terão contra-pressões (pontos de vista opostos) mais intensas nas decisões sobre a ideia social do que nas decisões sobre os produtos convencionais", as estratégias trabalham justamente com o oposto disso ao utilizar o incentivo por parte dos amigos e conhecidos para que a doação se realize, indo ao lado contrário da contrapressão e diminuindo-a. 
Causas sociais e as novas plataformas de comunicação: Teorias, viabilidade e práticas de Leandro Leonardo Batista e Nathalia Santos Andrijic

Para completar, as ações receberam reverberação a partir de ações de Relações Públicas da equipe de impresanos veículos locais, o que fez com que a comunidade tomasse um contato maior com a ideia e as ferramentas disponíveis.

\section{TEORIA E PRÁTICA: ANÁLISE DA PESQUISA EXPLORATÓRIA}

Todo o arcabouço teórico traçado suporta um entendimento mais aprofundado das estratégias utilizadas na comunicação de causas sociais e nos habilita a traçar uma análise de iniciativas que se utilizem das novas plataformas de comunicação. Assim, podemos entender o comportamento do emissor e também a diferença na recepção, ou não, com relação a um trabalho de marca, puramente. Considera-se aqui que as causas são diretamente relacionadas a quem faz a promoção, e é possível ver as diferenças, vantagens e desvantagens, e principalmente levando-se em conta o referencial teórico estudado.

É trazida aqui uma discussão que busca responder à pergunta colocada no início do trabalho com relação às diferenças, ou a ausência delas, entre promover uma marca e uma causa.

Para esta comparação será usado primordialmente o referencial de Manrai e Gardner (1992) com sua 8 proposições comparativas.

\subsection{Contribuições de Manrai e Gardner (1992)}

As autoras traçam 8 diferenças essenciais entre ideias sociais e produtos convencionais, trazidas no início do projeto como um grande ponto de suporte para a teorização do assunto aqui tratado.

O passo aqui é justamente explorar essas 8 proposições a partir dos 2 casos estudados para compreendê-los de maneira mais aprofundada e traçar as devidas comparações.

\begin{tabular}{|l|l|l|}
\hline DIFERENÇAS & PROPOSIÇÕES & ANÁLISE DOS CASOS \\
\hline $\begin{array}{l}\text { 1. Benefícios } \\
\text { compartilhados: }\end{array}$ & $\begin{array}{l}\text { Proposição 1: a percepção dos } \\
\text { consumidores de benefícios } \\
\text { individuais (em comparação com os } \\
\text { benefícios sociais) seria menor para }\end{array}$ & $\begin{array}{l}\text { O único caso em que o benefício individual já } \\
\text { se mostra mais evidente é o de Dumb ways to } \\
\text { die; para o outro, o benefício é mais coletivo. }\end{array}$ \\
\hline
\end{tabular}


Causas sociais e as novas plataformas de comunicação: Teorias, viabilidade e práticas de Leandro Leonardo Batista e Nathalia Santos Andrijic

\begin{tabular}{|c|c|c|}
\hline & $\begin{array}{l}\text { ideias sociais do que para os } \\
\text { produtos convencionais; }\end{array}$ & \\
\hline $\begin{array}{l}\text { 2. Responsabilidades } \\
\text { compartilhadas: }\end{array}$ & $\begin{array}{l}\text { Proposição 2: a percepção dos } \\
\text { consumidores de responsabilidade } \\
\text { individual (em comparação com a } \\
\text { social) seria menor para ideias } \\
\text { sociais do que para os produtos } \\
\text { convencionais; }\end{array}$ & $\begin{array}{l}\text { No caso da doação de sangue, principalmente, } \\
\text { a responsabilidade individual tem baixa } \\
\text { percepção, já que a alta nos bancos de sangue é } \\
\text { um problema coletivo. }\end{array}$ \\
\hline 3. Benefícios retardados: & $\begin{array}{l}\text { Proposição 3: percepção dos } \\
\text { consumidores sobre o tempo dentro } \\
\text { do qual benefícios do consumo } \\
\text { serão realizados seria maior para } \\
\text { ideias sociais do que para os } \\
\text { produtos convencionais; }\end{array}$ & $\begin{array}{l}\text { Morte nos trilhos do trem pode parecer uma } \\
\text { realidade muito distante; doação de sangue tem } \\
\text { um benefício muito retardado. }\end{array}$ \\
\hline 4. Controlabilidade: & $\begin{array}{l}\text { Proposição 4: percepção dos } \\
\text { consumidores de seu controle sobre } \\
\text { o uso e as consequências do } \\
\text { consumo seria menor para ideias } \\
\text { sociais do que para os produtos } \\
\text { convencionais; }\end{array}$ & $\begin{array}{l}\text { A percepção de controle das consequências } \\
\text { para os } 2 \text { é baixa. }\end{array}$ \\
\hline 5. Intangibilidade: & $\begin{array}{l}\text { Proposição 5: percepção dos } \\
\text { consumidores da tangibilidade das } \\
\text { ideias sociais seria menor do que a } \\
\text { de produtos convencionais; }\end{array}$ & $\begin{array}{l}\text { Manter-se seguro perto dos trens, doar sangue a } \\
\text { um terceiro desconhecido constituem em ações } \\
\text { pouco tangíveis. }\end{array}$ \\
\hline 6. Complexidade: & $\begin{array}{l}\text { Proposição 6: percepção dos } \\
\text { consumidores da complexidade de } \\
\text { mensagens seria maior para as } \\
\text { ideias sociais do que para os } \\
\text { produtos convencionais; }\end{array}$ & $\begin{array}{l}\text { As } 2 \text { mensagens têm alto nível de } \\
\text { complexidade. }\end{array}$ \\
\hline 7. Reversibilidade: & 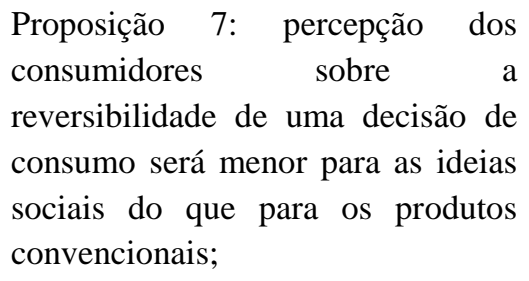 & $\begin{array}{l}\text { Identificar a percepção de reversibilidade é } \\
\text { complicado para os casos, já que as atitudes } \\
\text { tomadas não teriam a possibilidade de retorno. }\end{array}$ \\
\hline 8. Contra-pressões: & $\begin{array}{l}\text { Proposição 8: os consumidores } \\
\text { terão contra-pressões (pontos de } \\
\text { vista opostos) mais intensas nas } \\
\text { decisões sobre a ideia social do que } \\
\text { nas decisões sobre os produtos } \\
\text { convencionais. }\end{array}$ & $\begin{array}{l}\text { As contra-pressões são altas nos } 2 \text { casos, pela } \\
\text { falta de contribuição à segurança, e falta de } \\
\text { apoio à doação. }\end{array}$ \\
\hline
\end{tabular}


Causas sociais e as novas plataformas de comunicação: Teorias, viabilidade e práticas de Leandro Leonardo Batista e Nathalia Santos Andrijic

O mais interessante para observar no modelo de Manrai e Gardner (1992) são 3 aspectos do processamento da exposição a ideias de mensagens sociais: atenção, compreensão e afeição, envolvidos diretamente com as 8 proposições e diferenças. Assim, elas apresentam formas possíveis para minimizar esses efeitos trabalhando os 3 aspectos citados.

Os 4 primeiros pontos - benefícios compartilhados, responsabilidade compartilhada, benefícios retardados e controlabilidade - afetam a atenção para as mensagens. Assim, identifica-se um trabalho nos 2 casos para aumentar a atenção à mensagem. Dumb ways to die utiliza-se do entretenimento e de uma linguagem extremamente popular do jingle e da animação. Virginia Blood Services usa influenciadores locais para direcionar o foco de atenção

Para trabalhar as barreiras causadas pela intangibilidade e complexidade, as ações simplificam a atitude necessária aos envolvidos e as tangibilizam. Dumb ways to die com o uso da linguagem extremamente acessível; Virginia com os artifícios online para agendamento de doação.

Quanto às respostas afetivas, a reversibilidade e as contra-pressões foram endereçadas pela comunicação ao diminuir a associação do tema da morte nos trilhos com sentimentos desagradáveis com o compromisso; e diminuir contra-pressões sobre doação de sangue ao mostrar opiniões e casos.

Nos 2 casos, as 8 barreiras de Manrai e Gardner (1992) mostraram-se reais, assim como o trabalho em cima dos pontos de atenção, compreensão e afeição para quebrar essas barreiras.

\section{CONSIDERAÇÕES FINAIS}

As mídias sociais definitivamente já fazem parte do cotidiano de pessoas com os mais diversos interesses e condições e também das marcas, que buscam se apropriar das vantagens desses espaços e do contato que propiciam com seus públicos. Algumas promotoras de causas sociais também já identificaram essa oportunidade e estão se inserindo nesses comportamentos.

Com os 2 exemplos apresentados, Dumb ways to die e Virginia Blood Services, notou-se que as redes sociais têm sido eficientes para espalhar mensagens de campanhas sociais e levarem as pessoas a agirem. Tocam em aspectos essenciais para que a comunicação de causas sociais aconteça, com as melhores formas para diminuir os problemas relacionados a causas sociais e 
Causas sociais e as novas plataformas de comunicação: Teorias, viabilidade e práticas de Leandro Leonardo Batista e Nathalia Santos Andrijic

trabalhando com possibilidades das novas mídias ao seu favor.

Este projeto propôs-se a identificar as vantagens e desvantagens da adoção das estratégias do Marketing para a comunicação das causas sociais, visando compreender como o uso dessa abordagem poderia promover uma melhor transmissão de mensagens muitas vezes difíceis de serem comunicadas, por envolverem temáticas de difícil aproximação ou até mesmo muito esforço por parte do indivíduo, sendo que o benefício não é imediatamente perceptível ou é direcionado a terceiros. Apesar de desvantagens identificadas por um possível desvio de atenção ao real tema das campanhas ao aplicar-se estratégias de incentivo, prêmios e outras, a abordagem do Marketing se mostrou mais benéfica do que o contrário. Os modelos teóricos analisados e os casos estudados, provaram estar em sintonia.

A um dos questionamentos mais proeminentes da área, "Why can't you sell brotherhood and rational thinking like you sell soap?", de Wiebe em 1952, o presente estudo chega a conclusões que podem dar pistas de uma possível resposta. Foi entendido que na realidade não é possível 'vender causas sociais como se vende sabão', porque existem barreiras muito maiores em toda a relação das pessoas com as primeiras, mas que sim, a abordagem usada para o segundo pode trazer vantagens se bem aplicada às primeiras. Nas novas plataformas, principalmente, as possibilidades são férteis e a sua exploração para comunicar causas sociais é cada vez mais frequente, já que esses benefícios começam a ser percebidos.

Por fim, foram identificados diversos estudos sobre mídias sociais e as novas plataformas de comunicação, assim como sobre causas sociais e a abordagem do Marketing. Estudos relacionando as causas sociais em conjunção com as mídias sociais, porém, mostraram-se mais escassos, ainda que com uma tendência de crescimento nos anos mais recentes. O espaço para isso, e a necessidade do mercado, no entanto, são notórios, sendo muito bem-vindos estudos como o presente para identificar oportunidades de atuação e boas práticas que unam as novas plataformas com as causas sociais em prol de melhores e mais eficientes formas de comunicação.

\section{REFERÊNCIAS BIBLIOGRÁFICAS}

BIÇAKÇI, A. B. Persuading new generation consumers via Social Media: A case Study on Turkcell. Yeditepe University, 2012. Disponível em: <http://www.academia.edu/1610931/persuading_new_generation_consumers_via_social_ 
Causas sociais e as novas plataformas de comunicação: Teorias, viabilidade e práticas de Leandro Leonardo Batista e Nathalia Santos Andrijic

media_a_case_study_on_turkcell>.Acesso em: 24/12/2013

DIAZ, A. How 'Dumb Ways to Die' Won the Internet, Became the No. 1 Campaign of the Year. 2013. Disponível em: <http://adage.com/article/special-report-the-awards-report/dumbways-die-dissected/245195/>. Acesso em: 16/02/2014.

HANSON, C. L., MCKENZIE, J. F. NEIGER, B. L., THACKERAY, R. Enhancing Promotional Strategies Within Social Marketing Programs: Use of Web 2.0 Social Media. Health Promotion Practice. Vol. 9, No. 4, 2008: 338-343. Disponível em: <http://www.uk.sagepub.com/chaston/Chaston\%20Web\%20readings\%20chapters\%20112/Chapter\%209\%20-\%2031\%20Thackeray\%20et\%20al.pdf>. Acesso em: 17/10/2013

HASTINGS, G. HAYWOOD, A. Social marketing and communication in health promotion. Health Promotion International, 6(2), 1991: 135-145.

KAPLAN, A. M. \& HAENLEIN, M. Users of the world, unite! The challenges and opportunities of Social Media. Business Horizons, 53, 2010: 59-68. Disponível em: $<$ http://michaelhaenlein.com/Publications/Kaplan,\%20Andreas\%20\%20Users\%20of\%20the\%20world,\%20unite.pdf>. Acesso em 18/10/2013.

KOTLER, P., ZALTMAN G. Social Marketing: An approach to Planned Social Change. Journal of Marketing, 35 (July), 1971: 3-12.

MANGOLD, W. G., FAULDS, D. J. Social media: The new hybrid element of the promotion mix. . Business Horizons, 52, 2009: 357-365. Disponível em: <http://itu.dk/ rkva/2011Spring-EB22/readings/Mangold-SocialMedia.pdf>. Acesso em 22/12/2013.

MANRAI, L. A.; GARDNER, Meryl P. Consumer Processing of Social Ideas Advertising: a Conceptual Model In NA - Advances in Consumer Research Volume 19, eds. John F. Sherry, Jr. and Brian Sternthal, Provo, UT : Association for Consumer Research, 1992: 15-22. Disponível em: <http://www.acrwebsite.org/search/view-conferenceproceedings.aspx?Id=7262>. Acesso em: 26/12/2013.

WIEBE, G. D. Merchandising Commodities and Citizenship on Television. Public Opinion Quarterly 15, 1951-52: 679-91.

WIENER, J.; DOESCHER, T. A framework for promoting cooperation. Journal of Marketing, vol. $55,1991$.

Artigo submetido: 18/02/2015 
Causas sociais e as novas plataformas de comunicação: Teorias, viabilidade e práticas de Leandro Leonardo Batista e Nathalia Santos Andrijic

Artigo aprovado: 20/09/2015 